\title{
Fixed point theorems in preordered sets
}

\author{
Jarosław Górnicki®
}

\begin{abstract}
Ran and Reurings (Proc Am Math Soc 132(5):1435-1443, 2003) extended the Banach contraction principle to the setting of partially ordered metric spaces and recently Proinov (J Fixed Point Theory Appl 22:21, 2020) extended and unified many earlier fixed point theorems. In this paper we will present analogous results for the significantly wider class of mappings on preordered metric spaces. We give non-trivial examples of Kannan-type mappings.
\end{abstract}

Mathematics Subject Classification. Primary 47H10; Secondary 54H25.

Keywords. Asymptotic regularity, contractive mapping, complete metric space, fixed point, Kannan-type mapping, monotone mapping, $(\psi-$ $\varphi)$-type contraction, $(\psi-\varphi)$-type generalized contraction, preordered set.

\section{Introduction}

Ran and Reurings [16] established a fixed point theorem that extends the Banach contraction principle to the setting of partially ordered metric spaces. In the original version, Ran and Reurings used a continuous function. Next, Nieto and Rodríguez-López [11] established a similar result replacing the continuity of the nonlinear operator by monotonicity. These studies have been continued by many authors, see for example $[4,12]$.

The key feature in this theorem is that the contractivity condition on the nonlinear map is only assumed to hold on elements that are comparable in the partial order.

In this paper, we present the extension of the existing results to $(\psi-\varphi)$ type contractions, $(\psi-\varphi)$-type generalized contractions and and Kannan-type mappings on preordered spaces where a preordered binary relation is weaker than the partial order.

Let $(X, d)$ be a metric space and $T$ be a selfmap of $X$ satisfying the following type condition

$$
d(T x, T y) \leqslant K \cdot\{d(x, T x)+d(y, T y)\}
$$

for all $x, y \in X$, where $0 \leqslant K<\infty$ are fixed. 
Condition (1) does not ensure the existence of a fixed point [7, Example 1.1]. The situation changes when $T$ is additionally an asymptotically regular mapping, i.e., $\lim _{n \rightarrow \infty} d\left(T^{n+1} x, T^{n} x\right)=0$ for all $x \in X$.

Despite this the set of asymptotically regular mappings satisfying the condition (1) is rich. It includes Banach's contractions, Kannan's mappings with $K<\frac{1}{2}$ (not necessarily continuous) and many more, see $[5,9,17]$.

Example 1.1. Let $X=[0,2]$ and $d$ be the usual metric on $X$. Define $T: X \rightarrow$ $X$ by $T x=1$ if $0 \leqslant x \leqslant 1$ and $T x=0$ if $1<x \leqslant 2$. $T$ is not a continuous mapping. If $x, y \in[0,1]$ or $x, y \in(1,2]$, then $d(T x, T y)=0$. If $x \in[0,1]$ and $y \in(1,2]$, then $d(T x, T y)=1$ and $d(x, T x)+d(y, T y)=1-x+y>$ $2-x \geqslant 1$. Therefore $T$ satisfies (1) with $K=1$ and $M=0$. Of course $T$ is asymptotically regular.

Remark 1.2. If $T$ satisfies

$$
d(T x, T y) \leqslant M \cdot d(x, y)+K \cdot\{d(x, T x)+d(y, T y)\}
$$

for all $x, y \in X$, where $0<M<1$ and $0 \leqslant K<\infty$ (see [14, Example 4.5]), then $T$ satisfies

$$
d(T x, T y) \leqslant \frac{K+1}{1-M}\{d(x, T x)+d(y, T y)\}
$$

for all $x, y \in X$.

The situation is different when $M=1$. This is illustrated in the example below.

Example 1.3. Fix an $\varepsilon>0$. Let $X=[0, \infty)$ be with usual metric and $T$ : $X \rightarrow X$ be a mapping defined by $T x=0$ for $x \in[0, \infty) \cap \mathbb{Q}$ and $T x=x+\varepsilon$ for $x \in[0, \infty) \cap(\mathbb{R} \backslash \mathbb{Q})$.

$T$ does not satisfy (1) on $X$, there is no constant $K \in[0, \infty)$ such that for all $x, y \geqslant 0$ satisfying $|T x-T y| \leqslant K \cdot\{|x-T x|+|y-T y|\}$. Take $x=0$ and $y \in[0, \infty) \cap(\mathbb{R} \backslash \mathbb{Q})$, then any such $y \leqslant(K-1) \varepsilon$, and this is impossible. Also,

$$
|T x-T y|>\max \left\{|x-y|,|x-T x|,|y-T y|, \frac{1}{2}\{|x-T y|+|y-T x|\}\right\},
$$

take $x=0$ and $y=\sqrt{2}$, so $T$ does not satisfy Ćirić's condition and of course $T$ is not a Lipschitz mapping. Moreover, $T$ is not an asymptotically regular mapping. Let $\varepsilon \in(0, \infty) \cap(\mathbb{R} \backslash \mathbb{Q})$. Then $T^{n}(\varepsilon)=(n+1) \varepsilon$ and $\left|T^{n+1}(\varepsilon)-T^{n}(\varepsilon)\right| \rightarrow \varepsilon>0$ as $n \rightarrow \infty$.

On the other hand, $T$ satisfy

$$
|T x-T y| \leqslant|x-y|+\{|x-T x|+|y-T y|\}
$$

for all $x, y \geqslant 0$.

Indeed,

- if $x, y \geqslant 0$ are irrational numbers, then $|T x-T y|=|x-y|$ and the inequality is satisfied

$$
|T x-T y| \leqslant|x-y|+\{|x-T x|+|y-T y|\}=|x-y|+2 \varepsilon ;
$$


- if $x, y \geqslant 0$ are rational numbers, then the inequality is satisfied $|T x-T y|=0 \leqslant|x-y|+\{|x-T x|+|y-T y|\}=|x-y|+x+y ;$

- if $x, y \geqslant 0$ and $x$ is rational and $y$ is irrational, then the inequality is satisfied

$|T x-T y|=y+\varepsilon \leqslant|x-y|+\{|x-T x|+|y-T y|\}=|x-y|+x+\varepsilon$.

So it makes sense to study the mappings described using the control functions (see below).

We will now recall the necessary concepts.

Definition 1.4. Let $X \neq \emptyset$ be a set. Binary relation $\preccurlyeq$ on $X$ is

(a) reflexive if $x \preccurlyeq x$ for all $x \in X$,

(b) transitive if $x \preccurlyeq z$ for all $x, y, z \in X$ such that $x \preccurlyeq y$ and $y \preccurlyeq z$.

A reflexive and transitive relation on $X$ is a preordered on $X$. In such a case $(X, \preccurlyeq)$ is a preordered space. Write $x \prec y$ when $x \preccurlyeq y$ and $x \neq y$.

Example 1.5. (a) Let $\preccurlyeq$ be the binary relation on $\mathbb{R}$ given by

$$
x \preccurlyeq y \Leftrightarrow \quad(x=y \text { or } 0 \leqslant x<y) .
$$

Then $\preccurlyeq$ is a partial order (and so preordered) on $\mathbb{R}$, but is different from $\leqslant$.

(b) Let $X=C([a, b], \mathbb{R})$ with the usual metric $d(f, g)=\max _{t \in[a, b]} \mid f(t)-$ $g(t) \mid$. Consider on $X$ the partial order $\preccurlyeq$ defined by

$$
f, g \in X, \quad f \preccurlyeq g \Leftrightarrow \quad \forall_{t \in[a, b]} f(t) \leqslant g(t) .
$$

Then $(X, d, \preccurlyeq)$ is a partially ordered and complete metric space.

Definition 1.6. An preordered metric space is a triple $(X, d, \preccurlyeq)$ where $(X, d)$ is a metric space and $\preccurlyeq$ is a preorder on $X$.

One of the most important hypotheses that we shall use in next section is the monotonicity of the involved mappings.

Definition 1.7. Let $\preccurlyeq$ be a binary relation on $X$ and $T: X \rightarrow X$ be a mapping. We say that $T$ is $\preccurlyeq$-non-decreasing if $T x \preccurlyeq T y$ for all $x, y \in X$ such that $x \preccurlyeq y$.

After the appearance of the Ran and Reurings' result [16], Nieto and Rodríguez-López [11] changed the continuity of the mapping $T$ with the condition non-decreasing regularity.

Definition 1.8. Let $(X, d)$ be a metric space, let $A \subset X$ be a non-empty subset and let $\preccurlyeq$ be a binary relation on $X$. The triple $(X, d, \preccurlyeq)$ is said to be non-decreasing regular if for all sequence $\left\{x_{n}\right\} \subset A$ such that $x_{n} \rightarrow x \in A$ and $x_{n} \preccurlyeq x_{n+1}$ for all $n \in \mathbb{N}$, we have $x_{n} \preccurlyeq x$ for all $n \in \mathbb{N}$.

For example, the triple $(C[0,1), d, \preccurlyeq)$ from Example $1.5(\mathrm{~b})$ is non-decreasing regular. 


\section{A fixed point theorem for $(\psi-\varphi)$-type contraction}

In this section we extend some fixed point theorems established by Ran and Reurings [16] and Nieto and Rodrígez-López [11] and O'Regan and Petruşel [12] and the author [4] to mappings with the control functions on preordered metric spaces, where a preordered binary relation is weaker than a partial order.

Ćirić [2] introduced the notion of orbital continuity. If $T$ is a selfmapping of a metric space $(X, d)$ then the set $O(T, x)=\left\{T^{n} x: n=\right.$ $0,1,2, \ldots\}$ is called the orbit of $T$ at $x$ and $T$ is called orbitally continuous at a point $z \in X$ if for any sequence $\left\{x_{n}\right\} \subset O(T, x)$ for some $x \in X$, $x_{n} \rightarrow z$ implies $T x_{n} \rightarrow T z$ as $n \rightarrow \infty$.

Continuity of $T$ obviously implies orbital continuity but not conversely, $[2]$.

The weaker form of continuity is as follows [13]: a self-mapping $T$ of a metric space $(X, d)$ is called $k$-continuous, $k=1,2, \ldots$, if $T^{k} x_{n} \rightarrow T z$ whenever $\left\{x_{n}\right\}$ is a sequence in $X$ such that $T^{k-1} x_{n} \rightarrow z$ as $n \rightarrow \infty$.

It is easy to see that 1 -continuity is equivalent to continuity and

$$
\text { continuity } \Rightarrow 2-\text { continuity } \Rightarrow 3-\text { continuity } \Rightarrow \ldots \text {, }
$$

but not conversely. Also continuity of $T^{k}$ and $k$-continuity of $T$ are independent conditions when $k>1$, see [13, Examples 1.2-1.5].

We will begin our discussion when a mapping $T$ meets the classic contraction conditions. The starting point will be the following observation.

Lemma 2.1. [15, Lemma 2.1] Let $(X, d)$ be a metric space and $\left\{x_{n}\right\}$ be a sequence in $X$ which is not a Cauchy and $\lim _{n \rightarrow \infty} d\left(x_{n+1}, x_{n}\right)=0$. Then there exists $\varepsilon>0$ and two subsequences $\left\{x_{n_{k}}\right\}$ and $\left\{x_{m_{k}}\right\}$ of $\left\{x_{n}\right\}$ such that

$$
\lim _{k \rightarrow \infty} d\left(x_{n_{k}+1}, x_{m_{k}+1}\right)=\varepsilon+,
$$

and

$$
\lim _{k \rightarrow \infty} d\left(x_{n_{k}}, x_{m_{k}}\right)=\lim _{k \rightarrow \infty} d\left(x_{n_{k}+1}, x_{m_{k}}\right)=\lim _{k \rightarrow \infty} d\left(x_{n_{k}}, x_{m_{k}+1}\right)=\varepsilon .
$$

We will use this result in the proof of the following theorem, which requires only the fulfillment of the conditions on the set of comparable points, that is, for all $x, y \in X$ such that $x \preccurlyeq y$. In this way we will transfer some Proinov's results [15] to preordered sets.

Theorem 2.2. Let $(X, d, \preccurlyeq)$ be a preordered complete metric space and $T$ : $X \rightarrow X$ be a monotone ( $\preccurlyeq$-non-decreasing) mapping and such that $x_{0} \preccurlyeq T x_{0}$ for some $x_{0} \in X$, and

$$
\psi(d(T x, T y)) \leqslant \varphi(d(x, y))
$$

for any $x, y \in X$ with $x \preccurlyeq y$ and $d(T x, T y)>0$, where the functions $\psi, \varphi$ : $(0, \infty) \rightarrow \mathbb{R}$ satisfy the the following conditions:

(i) $\psi$ is non-decreasing;

(ii) $\varphi(t)<\psi(t)$ for any $t>0$;

and one of the following conditions: 
$\left(\right.$ iii $\left._{1}\right) \lim \sup _{t \rightarrow \varepsilon+} \varphi(t)<\psi(\varepsilon+)$ for any $\varepsilon>0$;

(iii $)) \lim \sup _{t \rightarrow \varepsilon} \varphi(t)<\liminf \operatorname{in}_{t \rightarrow \varepsilon+} \psi(t)$ for any $\varepsilon>0$;

(iii $\left.i_{3}\right) \lim \sup _{t \rightarrow \varepsilon+} \varphi(t)<\liminf _{t \rightarrow \varepsilon} \psi(t)$ for any $\varepsilon>0$.

Suppose that one of the following conditions holds:

(a) $T$ is $k$-continuous for some $k \geqslant 1$;

(b) $T$ is orbitally continuous,

(c) $(X, d, \preccurlyeq)$ is non-decreasing regular

Then $T$ has a fixed point.

If for every $(x, y) \in X \times X$ there exists $w \in X$ such that $x \preccurlyeq w$ and $y \preccurlyeq w$, we obtain uniqueness of the fixed point, say z. Moreover, for each $x_{0} \in X$ such that $x_{0} \preccurlyeq T x_{0}$, the sequence $\left\{T^{n} x_{0}\right\}$ of iterates converges to $z$.

Proof. Let $x_{0} \in X$ be a point satisfying $x_{0} \preccurlyeq T x_{0}$. We define a sequence $\left\{x_{n}\right\} \subset X$ as follows

$$
x_{n}=T x_{n-1}, \quad n \geqslant 1 .
$$

Regarding that $T$ is monotone mapping together with (5), we have

$$
x_{0} \preccurlyeq T x_{0}=x_{1} \quad \text { implies } x_{1}=T x_{0} \preccurlyeq T x_{1}=x_{2} \text {. }
$$

Inductively, we obtain

$$
x_{0} \preccurlyeq x_{1} \preccurlyeq x_{2} \preccurlyeq \cdots \preccurlyeq x_{n-1} \preccurlyeq x_{n} \preccurlyeq x_{n+1} \preccurlyeq \cdots
$$

Note that the sequence $\left\{x_{n}=T x_{n-1}\right\}$ is asymptotically regular.

Indeed, set $d_{n}=d\left(x_{n+1}, x_{n}\right)$ for $n \geqslant 0$. We will prove that $d_{n} \rightarrow 0$ as $n \rightarrow \infty$. It is trivial if $d_{n}=0$ for some $n \geqslant 0$. Suppose $d_{n}>0$ for every $n \geqslant 0$. Applying (4) and (ii), we obtain

$$
\psi\left(d_{n+1}\right) \leqslant \varphi\left(d_{n}\right)<\psi\left(d_{n}\right) .
$$

Then it follows from (6) and (i) that $d_{n+1}<d_{n}$ for every $n \geqslant 0$. Hence, the sequence $\left\{d_{n}\right\}$ is positive, strictly decreasing and it is bounded below. This implies that there exists $d \geqslant 0$ such that $d_{n} \rightarrow d+$ as $n \rightarrow \infty$.

Assume that $d>0$ and $\psi$ and $\varphi$ satisfy ( iii $\left._{1}\right)$. Taking the limit superior as $n \rightarrow \infty$ in the first inequality (6), we obtain

$$
\psi(d+)=\lim _{n \rightarrow \infty} \psi\left(d_{n+1}\right) \leqslant \limsup _{n \rightarrow \infty} \varphi\left(d_{n}\right) \leqslant \limsup _{d \rightarrow d+} \varphi(t),
$$

which is a contradiction to $\left(\mathrm{iii}_{1}\right)$. Hence, $\lim _{n \rightarrow \infty} d\left(x_{n}, x_{n+1}\right)=0$.

Assume that $d>0$ and $\psi$ and $\varphi$ satisfy ( iii $\left._{2}\right)$. Since $d_{n} \rightarrow d+$ as $n \rightarrow \infty$, so taking the limit as $n \rightarrow \infty$ in the first inequality (6), we obtain

$$
\liminf _{t \rightarrow d+} \psi(t) \leqslant \liminf _{n \rightarrow \infty} \psi\left(d_{n+1}\right) \leqslant \limsup _{n \rightarrow \infty} \varphi\left(d_{n}\right) \leqslant \limsup _{t \rightarrow d} \varphi(t),
$$

which is a contradiction to $\left(\mathrm{iii}_{2}\right)$. Hence, $\lim _{n \rightarrow \infty} d\left(x_{n}, x_{n+1}\right)=0$.

Assume that $d>0$ and $\psi$ and $\varphi$ satisfy ( iii $\left._{3}\right)$. Since $d_{n} \rightarrow d+$ as $n \rightarrow \infty$, so taking the limit as $n \rightarrow \infty$ in the first inequality (6), we obtain

$$
\liminf _{t \rightarrow d} \psi(d) \leqslant \liminf _{n \rightarrow \infty} \psi\left(d_{n+1}\right) \leqslant \limsup _{n \rightarrow \infty} \varphi\left(d_{n}\right) \leqslant \limsup _{t \rightarrow d+} \varphi(t),
$$

which is a contradiction to $\left(\mathrm{iii}_{3}\right)$. Hence, $\lim _{n \rightarrow \infty} d\left(x_{n}, x_{n+1}\right)=0$. 
If $x_{n}=x_{n+1}$ for some $n \geqslant 0$, then $x_{n}=T x_{n}$, so $x_{n}$ is the fixed point of $T$.

Suppose, $x_{n} \neq x_{n+1}$ for all $n \geqslant 0$. Assume that $\left\{x_{n}\right\}$ is not a Cauchy sequence.

Let $\psi$ and $\varphi$ satisfy $\left(\right.$ iii $\left._{1}\right)$. It follows from Lemma 2.1 that there exists $\varepsilon>0$ and two subsequences $\left\{x_{n_{k}}\right\}$ and $\left\{x_{m_{k}}\right\}$ of $\left\{x_{n}\right\}$ such that the limits (2) and (3) hold. It follows from (2) that $d\left(x_{n_{k}+1}, x_{m_{k}+1}\right)>\varepsilon$ for all $k \geqslant 1$. Applying (4) with $x=x_{n_{k}}$ and $y=x_{m_{k}}$, we get

$$
\psi\left(d\left(x_{n_{k}+1}, x_{m_{k}+1}\right)\right) \leqslant \varphi\left(d\left(x_{n_{k}}, x_{m_{k}}\right)\right)
$$

for all $k \geqslant 1$. Hence, taking into account that $\varphi(t)<\psi(t)$, we obtain

$$
\psi\left(d\left(x_{n_{k}+1}, x_{m_{k}+1}\right)\right) \leqslant \varphi\left(d\left(x_{n_{k}}, x_{m_{k}}\right)\right)<\psi\left(d\left(x_{n_{k}}, x_{m_{k}}\right)\right) .
$$

From this and monotonicity of $\psi$, we deduce that $d\left(x_{n_{k}+1}, x_{m_{k}+1}\right)<d$ $\left(x_{n_{k}}, x_{m_{k}}\right)$. Then it follows from (2) and (3) that $d\left(x_{n_{k}+1}, x_{m_{k}+1}\right) \rightarrow \varepsilon+$ and $d\left(x_{n_{k}}, x_{m_{k}}\right) \rightarrow \varepsilon+$ as $k \rightarrow \infty$. Taking the limit superior as $k \rightarrow \infty$ in (7), we get

$$
\begin{aligned}
\psi(\varepsilon+) & =\lim _{k \rightarrow \infty} \psi\left(d\left(x_{n_{k}+1}, x_{m_{k}+1}\right)\right) \\
& \leqslant \limsup _{k \rightarrow \infty} \varphi\left(d\left(x_{n_{k}}, x_{m_{k}}\right)\right) \\
& \leqslant \limsup _{t \rightarrow \varepsilon+} \varphi(t),
\end{aligned}
$$

which is a contradiction to $\left(\right.$ iii $\left._{1}\right)$.

Let $\psi$ and $\varphi$ satisfy $\left(\mathrm{iii}_{2}\right)$. Then, similarly to the previous case from (7), we get

$$
\begin{aligned}
\liminf _{t \rightarrow \varepsilon+} \psi(t) & \leqslant \liminf _{k \rightarrow \infty} \psi\left(d\left(x_{n_{k}+1}, x_{m_{k}+1}\right)\right) \leqslant \\
& \leqslant \limsup _{k \rightarrow \infty} \varphi\left(d\left(x_{n_{k}}, x_{m_{k}}\right) \leqslant\right. \\
& \leqslant \limsup _{t \rightarrow \varepsilon} \varphi(t),
\end{aligned}
$$

which is a contradiction to ( (iii $\left._{2}\right)$.

Let $\psi$ and $\varphi$ satisfy $\left(\mathrm{iii}_{3}\right)$. As in Lemma 2.1 (see [15, Lemma 2.2]) there exists $\varepsilon>0$ and two subsequences $\left\{x_{n_{k}}\right\}$ and $\left\{x_{m_{k}}\right\}$ of $\left\{x_{n}\right\}$ such that $\lim _{k \rightarrow \infty} d\left(x_{n_{k}}, x_{m_{k}}\right)=\varepsilon+$ and $\lim _{k \rightarrow \infty} d\left(x_{n_{k}+1}, x_{m_{k}+1}\right)=\varepsilon$. Hence, we conclude that $d\left(T x_{n_{k}}, T x_{m_{k}}\right)=d\left(x_{n_{k}+1}, x_{m_{k}+1}\right)>0$ and inequality (7) hold for infinitely many values of $k$. Then

$$
\begin{aligned}
\liminf _{t \rightarrow \varepsilon} \psi(t) & \leqslant \liminf _{k \rightarrow \infty} \psi\left(d\left(x_{n_{k}+1}, x_{m_{k}+1}\right)\right) \leqslant \\
& \leqslant \limsup _{k \rightarrow \infty} \varphi\left(d\left(x_{n_{k}}, x_{m_{k}}\right) \leqslant\right. \\
& \leqslant \limsup _{t \rightarrow \varepsilon+} \varphi(t),
\end{aligned}
$$

which is a contradiction to $\left(\mathrm{iii}_{3}\right)$.

Therefore, in any case, $\left\{x_{n}=T^{n} x_{0}\right\}$ is a Cauchy sequence. Because $X$ is complete $\lim _{n \rightarrow \infty} x_{n}=z \in X$. 
Part (a). Suppose that $T$ is $k$-continuous. Since $T^{k-1} x_{n} \rightarrow z, k$-continuity of $T$ implies that $T^{k} x_{n} \rightarrow T z$ as $n \rightarrow \infty$. By uniqueness of the limit (for each $j \geqslant 1$ we have, $T^{j} x_{n} \rightarrow z$ as $\left.n \rightarrow \infty\right), z=T z$.

Part (b). Suppose that $T$ is orbitally continuous. Since $x_{n} \rightarrow z$, orbital continuity implies that $T x_{n} \rightarrow T z$ as $n \rightarrow \infty$. This yields, by uniqueness of the limit, $z=T z$.

Part $(c)$. Due to assumption of non-decreasing regularity, we have $x_{n} \preccurlyeq z$ for all $n \geqslant 0$.

If $d\left(T^{n} x_{0}, T z\right)=0$ for infinitely many values of $n$, then

$$
d(z, T z) \leqslant d\left(z, T^{n} x_{0}\right)+d\left(T^{n} x_{0}, T z\right)=d\left(z, T^{n} x_{0}\right)
$$

for these values of $n$. Taking the limit as $n \rightarrow \infty$, we get $d(z, T z) \leqslant 0$ which implies $z=T z$.

Suppose $d\left(T^{n} x_{0}, T z\right)>0$ holds for infinitely many values of $n$. Then applying (4), we have

$$
\psi\left(d\left(T^{n+1} x_{0}, T z\right)\right) \leqslant \varphi\left(d\left(T^{n} x_{0}, z\right)\right)
$$

holds for these values of $n$. Since $\psi$ and $\varphi$ satisfy condition (ii), by (8), we have

$$
\psi\left(d\left(T^{n+1} x_{0}, T z\right)\right) \leqslant \varphi\left(d\left(T^{n} x_{0}, z\right)\right)<\psi\left(d\left(T^{n} x_{0}, z\right)\right) .
$$

From this and monotonicity of $\psi$, we have $d\left(T^{n+1} x_{0}, T z\right)<d\left(T^{n} x_{0}, z\right)$. Taking the limit as $n \rightarrow \infty$, we get $d(z, T z) \leqslant 0$. This means that $z=T z$.

To prove uniqueness, we assume that $v \in X$ is another fixed point of $T$ such that $v \neq z$. By hypothesis, there exists $w \in X$ such that $z \preccurlyeq w$ and $v \preccurlyeq w$.

Let $\left\{w_{n}=T w_{n-1}\right\}$ be the Picard sequence of $T$ based on $w_{0}=w$. As $T$ is $\preccurlyeq$-non-decreasing, $v=T v \preccurlyeq T w=w_{1}$ and $z=T z \preccurlyeq T w=w_{1}$. By induction, $v \preccurlyeq w_{n}$ and $z \preccurlyeq w_{n}$ for all $n \geqslant 0$.

Case 1. If $v=w_{n_{0}}$ for some $n_{0} \geqslant 0$, then $v=T v=T w_{n_{0}}=w_{n_{0}+1}$ and by induction, $w_{n}=v$ for all $n \geqslant n_{0}$, so $w_{n} \rightarrow v$ as $n \rightarrow \infty$.

Case 2. If $v \prec w_{n}$ for all $n \geqslant 0$, then $d\left(v, w_{n}\right)>0$ for all $n \geqslant 0$ and by (4) and (ii), we have

$$
\psi\left(d\left(v, w_{n+1}\right)\right)=\psi\left(d\left(T v, T w_{n}\right)\right) \leqslant \varphi\left(d\left(v, w_{n}\right)\right)<\psi\left(d\left(v, w_{n}\right)\right) .
$$

This implies that $d\left(v, w_{n+1}\right)<d\left(v, w_{n}\right)$ for $n \geqslant 0$. Hence the sequence $\left\{d\left(v, w_{n}\right)\right\}$ is positive, strictly decreasing and bounded below. This implies that there exists $d \geqslant 0$ such that $d\left(v, w_{n}\right) \rightarrow d+$ as $n \rightarrow \infty$. If $d>0$ we obtain a contradiction.

Let $\psi$ and $\varphi$ satisfy $\left(\mathrm{iii}_{1}\right)$. Taking the limit superior as $n \rightarrow \infty$ in the first part of inequality (9) we obtain

$$
\psi(d+)=\lim _{n \rightarrow \infty} \psi\left(d\left(v, w_{n+1}\right)\right) \leqslant \limsup _{n \rightarrow \infty} \varphi\left(d\left(v, w_{n}\right)\right) \leqslant \limsup _{t \rightarrow d+} \varphi(t),
$$

which is a contradiction to ( $\left.\mathrm{iii}_{1}\right)$. Hence, $w_{n} \rightarrow v$ as $n \rightarrow \infty$.

Let $\psi$ and $\varphi$ satisfy $\left(\right.$ iii $\left._{2}\right)$. Since $d\left(v, w_{n}\right) \rightarrow d+$ as $n \rightarrow \infty$, so taking the limit as $n \rightarrow \infty$ in the first inequality (9), we obtain

$$
\liminf _{t \rightarrow d+} \psi(t) \leqslant \liminf _{n \rightarrow \infty} \psi\left(d\left(v, w_{n+1}\right)\right) \leqslant \limsup _{n \rightarrow \infty} \varphi\left(d\left(v, w_{n}\right)\right) \leqslant \limsup _{t \rightarrow d} \varphi(t)
$$


which is a contradiction to ( iii $\left._{2}\right)$. Hence, $w_{n} \rightarrow v$ as $n \rightarrow \infty$.

Let $\psi$ and $\varphi$ satisfy $\left(\mathrm{iii}_{3}\right)$. Since $d\left(v, w_{n}\right) \rightarrow d+$ as $n \rightarrow \infty$, so taking the limit as $n \rightarrow \infty$ in the first inequality (9), we obtain

$$
\liminf _{t \rightarrow d} \psi(d) \leqslant \liminf _{n \rightarrow \infty} \psi\left(d\left(v, w_{n+1}\right)\right) \leqslant \limsup _{n \rightarrow \infty} \varphi\left(d\left(v, w_{n}\right)\right) \leqslant \limsup _{t \rightarrow d+} \varphi(t),
$$

which is a contradiction to $\left(\mathrm{iii}_{3}\right)$. Hence, $w_{n} \rightarrow v$ as $n \rightarrow \infty$.

Thus, $w_{n} \rightarrow v$ and $w_{n} \rightarrow z$. The uniqueness of the limit concludes that $v=z$, so $T$ has a unique fixed point.

It is clear that for each $x_{0} \in X$ such that $x_{0} \preccurlyeq T x_{0}$, the sequence $\left\{T^{n} x_{0}\right\}$ converges to $z$.

Remark 2.3. (A) If $\psi(t)=t$ and $\varphi(t)=M \cdot t$, where $M \in[0,1)$, then Theorem 2.2 is a generalization of the Banach fixed point theorem (1922) and the Ran-Reurings fixed point theorem (2003) [16] and Nieto-RodríguezLópez theorem (2005) [11].

(B) If $\psi(t)=t$, then Theorem 2.2 is a generalization of Boyd-Wong's fixed point theorem (1969) [1].

(C) Taking $\varphi(t)=\alpha(t) \psi(t)$, where $\alpha:(0, \infty) \rightarrow(0,1)$ such that $\lim \sup _{t \rightarrow \varepsilon+}$ $\alpha(t)<1$ for any $\varepsilon>0$, we will get some generalization of Geraghty's theorem [3], [15, Corollary 3.15]. Especially in the case $\varphi(t)=\alpha(t) \cdot t$ we obtain well-known fixed point theorem of Geraghty (1973). Taking $\varphi(t)=M \cdot \psi(t), M \in(0,1)$, we get special case of Skof's fixed point theorem (1977), see [15, Corollary 3.17], [18].

(D) Let $\psi:(0, \infty) \rightarrow \triangle, \triangle \subset \mathbb{R}$ be an open interval and $F: \triangle \rightarrow \mathbb{R}$ be an upper semicontinuous function with $F(t)<t$ for all $t \in \triangle$. Taking $\varphi(t)=F(\psi(t))$ we obtain improvement of Moradi fixed point theorem (2014), see [15, Corollary 3.14] and the references given there.

(E) Setting $\varphi(t)=\psi(t)-\tau$ where $\tau>0$ we obtain improvement of Wardowski fixed point theorem (2012), see [15, Corollary 3.21] and the references given there. Note that the fixed point theorem of Wardowski and a special case of the fixed point theorem of Skof (see $(C)$ ) are equivalent [15, Theorem 3.23].

(F) Theorem 2.2 remains true if $\varphi(t)=\psi(t)-\phi(t)$, where $\psi:(0, \infty) \rightarrow \mathbb{R}$ and $\phi:(0, \infty) \rightarrow(0, \infty)$ are functions such that:

(i') $\psi$ is non-decreasing;

(ii') $\liminf \operatorname{in}_{t \rightarrow \varepsilon+} \phi(t)>0$ for any $\varepsilon>0$, see [15, Section 4] and the references given there.

Remark 2.4. The assumptions in Theorem 2.2 that ensure the existence of exactly one fixed point are important, see [11].

Remark 2.5. The presented theorems remain true in preordered dislocated metric spaces because we do not use axiom $d(x, x)=0$. Recall $(X, \widehat{d})$ is called a dislocated metric space if the following conditions are satisfied for all $x, y, z \in X$,

$$
\begin{aligned}
& \widehat{d}(x, y)=0 \text { implies } x=y, \\
& \widehat{d}(x, y)=\widehat{d}(y, x),
\end{aligned}
$$




$$
\widehat{d}(x, y) \leqslant \widehat{d}(x, z)+\widehat{d}(z, y) .
$$

The motivation of defining this notion was to get better results in logic programming semantics [8].

\section{A fixed point theorem for $(\psi-\varphi)$-type generalized contraction}

Now we provide a fixed point theorem for a self-mapping $T$ on a preordered complete metric space satisfying a generalized contractive-type condition. This theorem requires more restrictive assumptions.

Theorem 3.1. Let $(X, d, \preccurlyeq)$ be a preordered complete metric space and $T$ : $X \rightarrow X$ be a monotone ( $\preccurlyeq$-non-decreasing) mapping and such that $x_{0} \preccurlyeq T x_{0}$ for some $x_{0} \in X$, and

$$
\psi(d(T x, T y)) \leqslant \varphi(m(x, y)),
$$

where

$$
m(x, y)=\max \left\{d(x, y), d(x, T x), d(y, T y), \frac{1}{2}[d(x, T y)+d(y, T x)]\right\},
$$

for any $x, y \in X$ with $x \preccurlyeq y$ and $d(T x, T y)>0$, where the functions $\psi, \varphi$ : $(0, \infty) \rightarrow \mathbb{R}$ satisfy the the following conditions:

(i) $\psi$ is non-decreasing and bounded below $\left(\inf _{t>\varepsilon} \psi(t)>-\infty\right.$ for any $\varepsilon>$ $0)$;

(ii) $\varphi(t)<\psi(t)$ for any $t>0$;

(iii) $\lim \sup _{t \rightarrow \varepsilon+} \varphi(t)<\psi(\varepsilon+)$ for any $\varepsilon>0$.

Suppose that one of the following conditions holds:

(a) $T$ is $k$-continuous for some $k \geqslant 1$;

(b) $T$ is orbitally continuous,

(c) $(X, d, \preccurlyeq)$ is non-decreasing regular

Then $T$ has a fixed point.

If for every $(x, y) \in X \times X$ there exists $w \in X$ such that $x \preccurlyeq w$ and $y \preccurlyeq w$ and $\left\{T^{n} w\right\}$ is an asymptotically regular sequence, we obtain uniqueness of the fixed point, say $z$. Moreover, for each $x_{0} \in X$ such that $x_{0} \preccurlyeq T x_{0}$, the sequence $\left\{T^{n} x_{0}\right\}$ of iterates converges to $z$.

Proof. Following the proof of Theorem 2.2, we have a $\preccurlyeq$-non-decreasing sequence $\left\{x_{n}=T x_{n-1}\right\}, n \geqslant 1$ :

$$
x_{0} \preccurlyeq x_{1} \preccurlyeq x_{2} \preccurlyeq \cdots \preccurlyeq x_{n-1} \preccurlyeq x_{n} \preccurlyeq x_{n+1} \preccurlyeq \cdots
$$

Conditions (i)-(iii) imply that $T$ is asymptotically regular mapping. Let $d_{n}=d\left(x_{n}, x_{n+1}\right)$. We have to prove that $d_{n} \rightarrow 0$ as $n \rightarrow \infty$.

It is trivial if $d_{n}=0$ for some $n \geqslant 0$. Suppose $d_{n}>0$ for every $n \geqslant 0$. It follows from (10) that

$$
\psi\left(d_{n+1}\right) \leqslant \varphi\left(m\left(x_{n}, x_{n+1}\right)\right) .
$$

It is easy to show that $m\left(x_{n}, x_{n+1}\right)=\max \left\{d_{n}, d_{n+1}\right\}$. Hence, if $d_{n} \leqslant d_{n+1}$ for some $n \geqslant 0$, then $m\left(x_{n}, x_{n+1}\right)=d_{n+1}$. In this case condition (11) becomes 
$\psi\left(d_{n+1}\right) \leqslant \varphi\left(d_{n+1}\right)$ which is a contradiction to (ii). Consequently $d_{n+1}<d_{n}$ and $m\left(x_{n}, x_{n+1}\right)=d_{n}$ for all $n \geqslant 0$. The sequence $\left\{d_{n}\right\}$ is positive, strictly decreasing and bounded below, which implies that there exists $d \geqslant 0$ such that $d_{n} \rightarrow d+$ as $n \rightarrow \infty$. It remains to prove that $d=0$. Assume $d>0$. It follows from (11) and (ii) that

$$
\psi\left(d_{n+1}\right) \leqslant \varphi\left(d_{n}\right)<\psi\left(d_{n}\right) .
$$

From this we conclude that $\left\{\psi\left(d_{n}\right)\right\}$ is a decreasing sequence. It follows from the second part of (i), that $\left\{\psi\left(d_{n}\right)\right\}$ is bounded below since $d_{n}>d$. Therefore, $\left\{\psi\left(d_{n}\right)\right\}$ is a convergent sequence. In view of (12) the sequence $\left\{\varphi\left(d_{n}\right)\right\}$ is also convergent with the same limit. Then

$$
\psi(d+)=\lim _{n \rightarrow \infty} \psi\left(d_{n}\right)=\lim _{n \rightarrow \infty} \varphi\left(d_{n}\right) \leqslant \limsup _{t \rightarrow d+} \varphi(t),
$$

which is a contradiction to (iii). Hence, $\lim _{n \rightarrow \infty} d\left(x_{n}, x_{n+1}\right)=0$.

If $x_{n}=x_{n+1}$ for some $n \geqslant 0$, then $x_{n}=T x_{n}$, so $x_{n}$ is the fixed point of $T$.

Suppose, $x_{n} \neq x_{n+1}$ for all $n \geqslant 0$. Assume that $\left\{x_{n}\right\}$ is not a Cauchy sequence. It follows from Lemma 2.1 that there exists $\varepsilon>0$ and two subsequences $\left\{x_{n_{k}}\right\}$ and $\left\{x_{m_{k}}\right\}$ of $\left\{x_{n}\right\}$ such that the limits (2) and (3) hold. It follows from (2) that $d\left(x_{n_{k}+1}, x_{m_{k}+1}\right)>\varepsilon>0$ for all $k \geqslant 1$. Applying (10) with $x=x_{n_{k}}$ and $y=x_{m_{k}}$, we get

$$
\psi\left(d\left(x_{n_{k}+1}, x_{m_{k}+1}\right)\right) \leqslant \varphi\left(m\left(x_{n_{k}}, x_{m_{k}}\right)\right)
$$

for all $k \geqslant 1$. Hence, taking into account that $\varphi(t)<\psi(t)$, we obtain

$$
\psi\left(d\left(x_{n_{k}+1}, x_{m_{k}+1}\right)\right) \leqslant \varphi\left(m\left(x_{n_{k}}, x_{m_{k}}\right)\right)<\psi\left(m\left(x_{n_{k}}, x_{m_{k}}\right)\right) .
$$

From this and monotonicity of $\psi$, we have $d\left(x_{n_{k}+1}, x_{m_{k}+1}\right)<m\left(x_{n_{k}}, x_{m_{k}}\right)$. Then it follows from (2) and (3) that $d\left(x_{n_{k}+1}, x_{m_{k}+1}\right) \rightarrow \varepsilon+$ and $m\left(x_{n_{k}}, x_{m_{k}}\right)$ $\rightarrow \varepsilon+$. Taking the limit superior as $k \rightarrow \infty$ in (13), we get

$$
\begin{aligned}
\psi(\varepsilon+) & =\lim _{k \rightarrow \infty} \psi\left(d\left(x_{n_{k}+1}, x_{m_{k}+1}\right)\right) \leqslant \\
& \leqslant \limsup _{k \rightarrow \infty} \varphi\left(m\left(x_{n_{k}}, x_{m_{k}}\right)\right) \leqslant \\
& \leqslant \limsup _{t \rightarrow \varepsilon+} \varphi(t),
\end{aligned}
$$

which is a contradiction to (iii).

Therefore, $\left\{x_{n}=T^{n} x_{0}\right\}$ is a Cauchy sequence. Because $X$ is complete $\lim _{n \rightarrow \infty} x_{n}=z \in X$.

Part (a). Suppose that $T$ is $k$-continuous. Since $T^{k-1} x_{n} \rightarrow z, k$-continuity of $T$ implies that $T^{k} x_{n} \rightarrow T z$ as $n \rightarrow \infty$. By uniqueness of the limit (for each $j \geqslant 1$ we have, $T^{j} x_{n} \rightarrow z$ as $\left.n \rightarrow \infty\right), z=T z$.

Part (b). Suppose that $T$ is orbitally continuous. Since $x_{n} \rightarrow z$, orbital continuity implies that $T x_{n} \rightarrow T z$ as $n \rightarrow \infty$. This yields, by uniqueness of the limit, $z=T z$.

Part (c). Due to assumption of non-decreasing regularity, we have $x_{n} \preccurlyeq z$ for all $n \geqslant 0$. 
If $d\left(T^{n} x_{0}, T z\right)=0$ for infinitely many values of $n$, then

$$
d(z, T z) \leqslant d\left(z, T^{n} x_{0}\right)+d\left(T^{n} x_{0}, T z\right)=d\left(z, T^{n} x_{0}\right)
$$

for these values of $n$. Taking the limit as $n \rightarrow \infty$, we get $d(z, T z) \leqslant 0$ which implies $z=T z$.

Assume $d(z, T z)=\varepsilon>0$. It follows from $T^{n} x_{0} \rightarrow z$ as $n \rightarrow \infty$ that $d\left(T^{n} x_{0}, T z\right) \rightarrow d(z, T z)$, which implies that $d\left(T^{n} x_{0}, T z\right)>0$ holds for infinitely many values of $n$. Then applying (10), we have

$$
\psi\left(d\left(T^{n+1} x_{0}, T z\right)\right) \leqslant \varphi\left(m\left(T^{n} x_{0}, z\right)\right)
$$

holds for these values of $n$. By the definition $m\left(T^{n} x_{0}, z\right)$ equals the maximum of four quantities $d\left(T^{n} x_{0}, z\right), d\left(T^{n} x_{0}, T^{n+1} x_{0}\right), \frac{1}{2}\left[d\left(T^{n} x_{0}, T z\right)+d\left(z, T^{n+1} x_{0}\right)\right]$ and $d(z, T z)$. Taking into account that $d\left(T^{n} x_{0}, z\right) \rightarrow 0, d\left(T^{n} x_{0}, T^{n+1} x_{0}\right) \rightarrow$ $0, d\left(z, T^{n+1} x_{0}\right) \rightarrow 0$ and $d\left(T^{n} x_{0}, T z\right) \rightarrow d(z, T z)$ as $n \rightarrow \infty$, we deduce that $m\left(T^{n} x_{0}, z\right)=d(z, T z)$ for sufficiently large values of $n$. Then from (14) we obtain

$$
\psi\left(d\left(T^{n+1} x_{0}, T z\right)\right) \leqslant \varphi(d(z, T z))
$$

for infinitely many values of $n$. Taking the limit inferior as $n \rightarrow \infty$ in (15), we get

$$
\liminf _{t \rightarrow \varepsilon} \psi(t) \leqslant \liminf _{n \rightarrow \infty} \psi\left(d\left(T^{n} x_{0}, T z\right)\right) \leqslant \varphi(\varepsilon)
$$

which is a contradiction to (i)-(ii), because if the functions $\psi$ and $\varphi$ satisfy the conditions (i) and (ii), then $\varphi(\varepsilon)<\liminf _{t \rightarrow \varepsilon} \psi(t)$ for any $\varepsilon>0$. Hence, $d(z, T z)=0$. This means that $z=T z$.

To prove uniqueness, we assume that $v \in X$ is another fixed point of $T$ such that $v \neq z$. By hypothesis, there exists $w \in X$ such that $z \preccurlyeq w$ and $v \preccurlyeq w$.

Let $\left\{w_{n}=T w_{n-1}\right\}$ be the Picard sequence of $T$ based on $w_{0}=w$. As $T$ is $\preccurlyeq$-non-decreasing, $v=T v \preccurlyeq T w=w_{1}$ and $z=T z \preccurlyeq T w=w_{1}$. By induction, $v \preccurlyeq w_{n}$ and $z \preccurlyeq w_{n}$ for all $n \geqslant 0$.

Case 1. If $v=w_{n_{0}}$ for some $n_{0} \geqslant 0$, then $v=T v=T w_{n_{0}}=w_{n_{0}+1}$ and by induction, $w_{n}=v$ for all $n \geqslant n_{0}$, so $w_{n} \rightarrow v$ as $n \rightarrow \infty$.

Case 2. If $v \prec w_{n}$ for all $n \geqslant 0$, then $d\left(v, w_{n}\right)>0$ for all $n \geqslant 0$ and by (10) and (ii), we have

$$
\psi\left(d\left(v, w_{n+1}\right)\right)=\psi\left(d\left(T v, T w_{n}\right)\right) \leqslant \varphi\left(m\left(v, w_{n}\right)\right)<\psi\left(m\left(v, w_{n}\right)\right) .
$$

From this and monotonicity of $\psi$, we have

$$
d\left(v, w_{n+1}\right)<m\left(v, w_{n}\right)
$$

for all $n \geqslant 0$. By the definition $m\left(v, w_{n}\right)$ equals the maximum of four quantities $d\left(v, w_{n}\right), d(v, T v)=0, d\left(w_{n}, T w_{n}\right)=d\left(T^{n} w_{0}, T^{n+1} w_{0}\right) \rightarrow 0$ as $n \rightarrow n$ (by asymptotic regularity) and $\frac{1}{2}\left[d\left(v, w_{n+1}\right)+d\left(w_{n}, T v\right)\right]$. Hence for sufficiently large values of $n$,

$$
m\left(v, w_{n}\right)=\max \left\{d\left(v, w_{n}\right), \frac{1}{2}\left[d\left(v, w_{n+1}\right)+d\left(v, w_{n}\right)\right]\right\} .
$$


If $m\left(v, w_{n}\right)=\frac{1}{2}\left[d\left(v, w_{n+1}\right)+d\left(v, w_{n}\right)\right]$ then by (17),

$$
d\left(v, w_{n+1}\right)<\frac{1}{2}\left[d\left(v, w_{n+1}\right)+d\left(v, w_{n}\right)\right],
$$

So

$$
d\left(v, w_{n+1}\right)<d\left(v, w_{n}\right) .
$$

If $m\left(v, w_{n}\right)=d\left(v, w_{n}\right)$, then by (17),

$$
d\left(v, w_{n+1}\right)<d\left(v, w_{n}\right) .
$$

Consequently the sequence $\left\{d\left(v, w_{n}\right)\right\}$ is positive and for sufficiently large values of $n$ is strictly decreasing, which implies that there exists $p \geqslant 0$ such that $d\left(v, w_{n}\right) \rightarrow p+$ as $n \rightarrow \infty$. Assume $p>0$. It follows from (16), (17) and (ii),

$$
\psi\left(d\left(v, w_{n+1}\right)\right) \leqslant \varphi\left(m\left(v, w_{n}\right)\right)=\varphi\left(d\left(v, w_{n}\right)\right)<\psi\left(m\left(v, w_{n}\right)\right)
$$

for sufficiently large values of $n$. From this, we conclude that $\left\{\psi\left(d\left(v, w_{n}\right)\right\}\right.$ is a decreasing sequence for these values of $n$ and is bounded below since $d\left(v, w_{n}\right)>p$. Therefore $\left\{\psi\left(d\left(v, w_{n}\right)\right\}\right.$ is a convergent sequence. In view of (18) the sequence $\left\{\varphi\left(d\left(v, w_{n}\right)\right\}\right.$ is also convergent with the same limit. Then

$$
\psi(p+)=\lim _{n \rightarrow \infty} \psi\left(d\left(v, w_{n}\right)\right)=\lim _{n \rightarrow \infty} \varphi\left(d\left(v, w_{n}\right)\right) \leqslant \limsup _{t \rightarrow p+} \varphi(t),
$$

which is a contradiction to (iii). Hence, $d\left(v, w_{n}\right) \rightarrow 0$ as $n \rightarrow \infty$.

Thus, $w_{n} \rightarrow v$ and $w_{n} \rightarrow z$. The uniqueness of the limit concludes that $v=z$, so $T$ has a unique fixed point.

It is clear that for each $x_{0} \in X$ such that $x_{0} \preccurlyeq T x_{0}$, the sequence $\left\{T^{n} x_{0}\right\}$ converges to $z$.

Remark 3.2. In the above theorem, it is possible to test the thesis by considering the following functions

- $\varphi(t)=F(\psi(t)), \varphi(t)=M \cdot \psi(t), \varphi(t)=\psi(t)-\tau$ with appropriately selected assumptions, see [15, Corollary 5.9, 5.12 and Theorem 5.13] and references therein. In particular, fixed point theorems of Wardowski-Van Dung (2014) and Li-Jiang (2016) are special cases of Skof's generalized fixed point theorem;

- $\varphi(t)=\psi(t)-\phi(t)$ with appropriately selected assumptions, see $[15$, Section 6] and references therein.

\section{Fixed point theorems for Kannan-type mappings}

We can easily prove the following result for Kannan-type mappings satisfying (1) in preordered spaces, see [6, Theorems 3.5, 3.8].

Theorem 4.1. Let $(X, d, \preccurlyeq)$ be a preordered complete metric space and $T$ : $X \rightarrow X$ be an asymptotically regular and monotone ( $\preccurlyeq$-non-decreasing) mapping and such that $x_{0} \preccurlyeq T x_{0}$ for some $x_{0} \in X$, and satisfying

$$
d(T x, T y) \leqslant K \cdot\{d(x, T x)+d(y, T y)\}
$$


for any $x, y \in X$ with $x \preccurlyeq y$, where $0 \leqslant K<\infty$. Suppose that one of the following conditions holds:

(a) $T$ is $k$-continuous for some $k \geqslant 1$;

(b) $T$ is orbitally continuous;

(c) $(X, d, \preccurlyeq)$ is non-decreasing regular and $0 \leqslant K<1$.

Then $T$ has a fixed point.

If for every $(x, y) \in X \times X$ there exists $w \in X$ such that $x \preccurlyeq w$ and $y \preccurlyeq w$, we obtain uniqueness of the fixed point, say $z$. Moreover, for each $x_{0} \in X$ such that $x_{0} \preccurlyeq T x_{0}$, the sequence $\left\{T^{n} x_{0}\right\}$ of iterates converges to $z$.

Remark 4.2. The assumptions in Theorem 4.1 that ensure the existence of exactly one fixed point are important, see [6, Examples 3.9 and 3.10].

The key feature in this theorem is that the contractivity condition on the nonlinear map is only assumed to hold on elements that are comparable.

Example 4.3. Let $X=\{0,1,2,3,8\}$ be a set of non-negative integers with usual metric. Then $(X, d)$ is a complete metric space. Consider on $X$ the partial order

$$
x \preccurlyeq y \Leftrightarrow \quad(x=y \text { or } 1 \leqslant x<y) .
$$

Define $T: X \rightarrow X$ as follows: $T 0=0, T 1=2, T 2=3, T 3=8$ and $T 8=8$. $T$ is continuous, asymptotically regular and $T$ satisfies

$$
d(T x, T y)>\max \left\{d(x, y), d(x, T x), d(y, T y), \frac{1}{2}(d(x, T y)+d(y, T x))\right\},
$$

take $x=1$ and $y=3$. In particular, $T$ is not a nonexpansive mapping.

$T$ does not satisfy (1) on $X$, there is no constant $K \in[0, \infty)$ such that for all $x, y \in X$ satisfying $d(T x, T y) \leqslant K \cdot\{d(x, T x)+d(y, T y)\}$, take $x=0$ and $y=8$.

On the other hand, $d(T x, T y) \leqslant 6\{d(x, T x)+d(y, T y)\}$ for all $x \preccurlyeq y$, i.e., for all $x, y \in\{1,2,3,8\} \subset X$, where $\{1,2,3,8\} \neq X$.

Our another generalization of the last theorem is inspired by theorem of Geraghty [3,7].

Let $\mathcal{S}$ denote the class of those functions $\alpha:[0, \infty) \rightarrow[0,1)$ which satisfy the simple condition: $\alpha\left(t_{n}\right) \rightarrow 1 \Rightarrow t_{n} \rightarrow 0$, for example, $\alpha_{1}(t)=e^{-t}$, $\alpha_{2}(t)=\frac{1}{1+t}, t>0$. Generally we do not assume that $\alpha$ is continuous in any sense.

Theorem 4.4. Let $(X, d, \preccurlyeq)$ be a preordered complete metric space and $T$ : $X \rightarrow X$ be an asymptotically regular and monotone ( $\preccurlyeq$-non-decreasing) mapping and such that $x_{0} \preccurlyeq T x_{0}$ for some $x_{0} \in X$, and

$$
d(T x, T y) \leqslant \alpha(d(x, y)) \cdot d(x, y)+K \cdot\{d(x, T x)+d(y, T y)\}
$$

for any $x, y \in X$ with $x \preccurlyeq y$, where a function $\alpha \in \mathcal{S}$ and $0 \leqslant K<\infty$. Suppose that one of the following conditions holds:

(a) $T$ is $k$-continuous for some $k \geqslant 1$;

(b) $T$ is orbitally continuous;

(c) $(X, d, \preccurlyeq)$ is non-decreasing regular and $0 \leqslant K<1$. 
Then $T$ has a fixed point.

If for every $(x, y) \in X \times X$ there exists $w \in X$ such that $x \preccurlyeq w$ and $y \preccurlyeq w$, we obtain uniqueness of the fixed point, say z. Moreover, for each $x_{0} \in X$ such that $x_{0} \preccurlyeq T x_{0}$, the sequence $\left\{T^{n} x_{0}\right\}$ of iterates converges to $z$.

Proof. Following the proof of Theorem 2.2, we have a $\preccurlyeq$-non-decreasing sequence $\left\{x_{n}=T x_{n-1}\right\}, n \geqslant 1$ :

$$
x_{0} \preccurlyeq x_{1} \preccurlyeq x_{2} \preccurlyeq \cdots \preccurlyeq x_{n-1} \preccurlyeq x_{n} \preccurlyeq x_{n+1} \preccurlyeq \cdots
$$

If $x_{n}=x_{n+1}$ for some $n \geqslant 0$, then $x_{n}=T x_{n}$, so $x_{n}$ is the fixed point of $T$.

Suppose, $x_{n} \neq x_{n+1}$ for all $n \geqslant 0$. Assume that $\left\{x_{n}\right\}$ is not a Cauchy sequence. Then $\lim \sup _{n, m \rightarrow \infty} d\left(x_{n}, x_{m}\right)>0$. By the triangle inequality,

$$
d\left(x_{n}, x_{m}\right) \leqslant d\left(x_{n}, x_{n+1}\right)+d\left(x_{n+1}, x_{m+1}\right)+d\left(x_{m+1}, x_{m}\right),
$$

so by (19)

$$
\begin{aligned}
d\left(x_{n}, x_{m}\right) \leqslant & \alpha\left(d\left(x_{n}, x_{m}\right)\right) \cdot d\left(x_{n}, x_{m}\right) \\
& +(K+1) \cdot\left\{d\left(x_{n}, x_{n+1}\right)+d\left(x_{m}, x_{m+1}\right)\right\} .
\end{aligned}
$$

Under the assumption $\lim \sup _{n, m \rightarrow \infty} d\left(x_{n}, x_{m}\right)>0$, asymptotic regularity of $\left\{x_{n}\right\}$, the inequality

$$
\frac{d\left(x_{n}, x_{m}\right)}{d\left(x_{n}, x_{n+1}\right)+d\left(x_{m}, x_{m+1}\right)} \leqslant \frac{K+1}{1-\alpha\left(d\left(x_{n}, x_{m}\right)\right)}
$$

now implies

$$
\limsup _{n, m \rightarrow \infty} \frac{K+1}{1-\alpha\left(d\left(x_{n}, x_{m}\right)\right)}=+\infty
$$

from which

$$
\limsup _{n, m \rightarrow \infty} \alpha\left(d\left(x_{n}, x_{m}\right)\right)=1 .
$$

From the properties of the $\alpha$ function, $\limsup _{n, m \rightarrow \infty} d\left(x_{n}, x_{m}\right)=0$, which is a contradiction. Thus $\left\{x_{n}\right\}$ is a Cauchy sequence. Because $X$ is complete $\lim _{n \rightarrow \infty} x_{n}=z \in X$.

Part $(a)$ and $(b)$. Analogically as in the proof of Theorem 2.2, $z$ is a fixed point of $T$.

Part $(c)$. Due to assumption of non-decreasing regularity, we have $x_{n} \preccurlyeq z$ for all $n \geqslant 0$.

Since $T^{n} x_{0} \rightarrow z$ and $d\left(T^{n+1} x_{0}, T^{n} x_{0}\right) \rightarrow 0$ as $n \rightarrow \infty$, using that $T^{n} x_{0} \preccurlyeq z$ for all $n \in \mathbb{N}$, we get

$$
\begin{aligned}
d(T z, z) \leqslant & d\left(T z, T^{n+1} x_{0}\right)+d\left(T^{n+1} x_{0}, z\right) \\
\leqslant & \alpha\left(d\left(z, T^{n} x_{0}\right)\right) \cdot d\left(z, T^{n} x_{0}\right)+K\left\{d(z, T z)+d\left(T^{n} x_{0}, T^{n+1} x_{0}\right)\right\} \\
& +d\left(T^{n+1} x_{0}, z\right),
\end{aligned}
$$

So

$$
\begin{aligned}
(1-K) d(T z, z) \leqslant & \alpha\left(d\left(z, T^{n} x_{0}\right)\right) \cdot d\left(z, T^{n} x_{0}\right)+K d\left(T^{n} x_{0}, T^{n+1} x_{0}\right)+ \\
& +d\left(T^{n+1} x_{0}, z\right) \rightarrow 0
\end{aligned}
$$


as $n \rightarrow \infty$. In consequence, $d(T z, z)=0$. Hence $z=T z$.

To prove uniqueness, we assume that $v \in X$ is another fixed point of $T$ such that $v \neq z$. By hypothesis, there exists $w \in X$ such that $z \preccurlyeq w$ and $v \preccurlyeq w$.

Let $\left\{w_{n}=T w_{n-1}\right\}$ be the Picard sequence of $T$ based on $w_{0}=w$. As $T$ is $\preccurlyeq$-non-decreasing, $v=T v \preccurlyeq T w=w_{1}$ and $z=T z \preccurlyeq T w=w_{1}$. By induction, $v \preccurlyeq w_{n}$ and $z \preccurlyeq w_{n}$ for all $n \geqslant 0$.

Case 1. If $v=w_{n_{0}}$ for some $n_{0} \geqslant 0$, then $v=T v=T w_{n_{0}}=w_{n_{0}+1}$ and by induction, $w_{n}=v$ for all $n \geqslant n_{0}$, so $w_{n} \rightarrow v$ as $n \rightarrow \infty$.

Case 2. If $v \prec w_{n}$ for all $n \geqslant 0$, then by (19)

$$
\begin{aligned}
d\left(v, w_{n+1}\right) & =d\left(T v, T w_{n}\right) \leqslant \\
& \leqslant \alpha\left(d\left(v, w_{n}\right)\right) \cdot d\left(v, w_{n}\right)+K\left\{d(v, T v)+d\left(w_{n}, w_{n+1}\right)\right\}= \\
& =\alpha\left(d\left(v, w_{n}\right)\right) \cdot d\left(v, w_{n}\right)+K d\left(w_{n}, w_{n+1}\right) .
\end{aligned}
$$

Assume $w_{n} \nrightarrow v$, i.e., $\lim \sup _{n \rightarrow \infty} d\left(v, w_{n}\right)>0$. Under the asymptotic regularity of $\left\{w_{n}\right\}$, the inequality

$$
\begin{gathered}
d\left(v, w_{n+1}\right) \leqslant \alpha\left(d\left(v, w_{n}\right)\right) \cdot\left[d\left(v, w_{n+1}\right)+d\left(w_{n+1}, w_{n}\right)\right]+K d\left(w_{n}, w_{n+1}\right), \\
{\left[1-\alpha\left(d\left(v, w_{n}\right)\right)\right] \cdot d\left(v, w_{n+1}\right) \leqslant\left[K+\alpha\left(d\left(v, w_{n}\right)\right)\right] \cdot d\left(w_{n}, w_{n+1}\right),}
\end{gathered}
$$

now implies, $\lim \sup _{n \rightarrow \infty} \alpha\left(d\left(v, w_{n}\right)\right)=1$, so $\lim \sup _{n \rightarrow \infty} d\left(v, w_{n}\right)=0$, which is a contradiction. Hence, $w_{n} \rightarrow v$ as $n \rightarrow \infty$.

Thus, $w_{n} \rightarrow v$ and $w_{n} \rightarrow z$. The uniqueness of the limit concludes that $v=z$, so $T$ has a unique fixed point.

It is clear that for each $x_{0} \in X$ such that $x_{0} \preccurlyeq T x_{0}$, the sequence $\left\{T^{n} x_{0}\right\}$ converges to $z$.

The next existential result is inspired by theorem of Boyd and Wong [1]. A mapping $T$ satisfying $d(T x, T y) \leqslant \varphi(d(x, y))$ where $\varphi(t)<t$ for each $t>0$, may not posses a fixed point unless some additional condition is assumed on $\varphi$. Boyd and Wong [1] assumed $\varphi$ to be upper semi-continuous from the right.

Let $\mathcal{U}$ denote the class of all mappings $\varphi:[0, \infty) \rightarrow[0, \infty)$ satisfying

(i) $\varphi(t)<t$ for all $t>0$,

(ii) $\varphi$ is upper semi-continuous, that is $t_{n} \downarrow t \geqslant 0 \Rightarrow \lim _{\sup _{n \rightarrow \infty}} \varphi\left(t_{n}\right) \leqslant$ $\varphi(t)$.

Theorem 4.5. Let $(X, d, \preccurlyeq)$ be a preordered complete metric space and $T$ : $X \rightarrow X$ be an asymptotically regular and monotone ( $\preccurlyeq$-non-decreasing) mapping and such that $x_{0} \preccurlyeq T x_{0}$ for some $x_{0} \in X$, and

$$
d(T x, T y) \leqslant \varphi(d(x, y))+K \cdot\{d(x, T x)+d(y, T y)\} .
$$

for any $x, y \in X$ with $x \preccurlyeq y$, where a function $\varphi \in \mathcal{U}$ and $0 \leqslant K<\infty$. Suppose that one of the following conditions holds:

(a) $T$ is $k$-continuous for some $k \geqslant 1$;

(b) $T$ is orbitally continuous,

(c) $(X, d, \preccurlyeq)$ is non-decreasing regular and $0 \leqslant K<1$. 
Then $T$ has a fixed point.

Proof. Following the proof of Theorem 2.2, we have a $\preccurlyeq$-non-decreasing sequence $\left\{x_{n}=T x_{n-1}\right\}, n \geqslant 1$ :

$$
x_{0} \preccurlyeq x_{1} \preccurlyeq x_{2} \preccurlyeq \ldots \preccurlyeq x_{n-1} \preccurlyeq x_{n} \preccurlyeq x_{n+1} \preccurlyeq \ldots
$$

If $x_{n}=x_{n+1}$ for some $n \geqslant 0$, then $x_{n}=T x_{n}$, so $x_{n}$ is the fixed point of $T$.

Suppose, $x_{n} \neq x_{n+1}$ for all $n \geqslant 0$. Assume that $\left\{x_{n}\right\}$ is not a Cauchy sequence. Then there exists $\varepsilon>0$ and integers $n_{i}, m_{i} \in \mathbb{N}$ such that $m_{i}>$ $n_{i} \geqslant i$ and

$$
d\left(x_{n_{i}}, x_{m_{i}}\right) \geqslant \varepsilon \quad \text { for } i=1,2, \ldots
$$

Also, choosing $m_{i}$ small as possible, if may be assumed that

$$
d\left(x_{n_{i}}, x_{m_{i}-1}\right)<\varepsilon .
$$

Hence for each $i \in \mathbb{N}$, we have

$$
\varepsilon \leqslant d\left(x_{n_{i}}, x_{m_{i}}\right) \leqslant d\left(x_{n_{i}}, x_{m_{i}-1}\right)+d\left(x_{m_{i}-1}, x_{m_{i}}\right)<\varepsilon+d\left(x_{m_{i}-1}, x_{m_{i}}\right),
$$

and it follows from asymptotic regularity that

$$
\lim _{i \rightarrow \infty} d\left(x_{n_{i}}, x_{m_{i}}\right)=\varepsilon .
$$

(It also provides Lemma 2.1.) Next observe, that

$$
\begin{aligned}
d\left(x_{n_{i}}, x_{m_{i}}\right) \leqslant & d\left(x_{n_{i}}, x_{n_{i}+1}\right)+d\left(x_{n_{i}+1}, x_{m_{i}+1}\right)+d\left(x_{m_{i}+1}, x_{m_{i}}\right) \\
\leqslant & d\left(x_{n_{i}}, x_{n_{i}+1}\right) \\
& +\varphi\left(d\left(x_{n_{i}}, x_{m_{i}}\right)\right)+K \cdot\left\{d\left(x_{n_{i}}, x_{n_{i}+1}\right)+d\left(x_{m_{i}}, x_{m_{i}+1}\right)\right\} \\
& +d\left(x_{m_{i}+1}, x_{m_{i}}\right) \\
= & \varphi\left(d\left(x_{n_{i}}, x_{m_{i}}\right)\right)+(K+1) \cdot\left\{d\left(x_{n_{i}}, x_{n_{i}+1}\right)+d\left(x_{m_{i}}, x_{m_{i}+1}\right)\right\} .
\end{aligned}
$$

Letting $i \rightarrow \infty$, using asymptotic regularity and upper semi-continuity of $\varphi$, we obtain

$$
0<\varepsilon=\lim _{i \rightarrow \infty} d\left(x_{n_{i}}, x_{m_{i}}\right) \leqslant \limsup _{i \rightarrow \infty} \varphi\left(d\left(x_{n_{i}}, x_{m_{i}}\right)\right) \leqslant \varphi(\varepsilon)<\varepsilon,
$$

which is a contradiction. Hence $\left\{x_{n}\right\}$ is a Cauchy sequence. Because $X$ is complete $\lim _{n \rightarrow \infty} x_{n}=z \in X$.

Part $(a)$ and (b). Analogically as in the proof of Theorem 2.2, $z$ is a fixed point of $T$.

Part (c). Due to assumption of non-decreasing regularity, we have $x_{n} \preccurlyeq z$ for all $n \geqslant 0$.

Since $T^{n} x_{0} \rightarrow z$ and $d\left(T^{n+1} x_{0}, T^{n} x_{0}\right) \rightarrow 0$ as $n \rightarrow \infty$, using that $T^{n} x_{0} \preccurlyeq z$ for all $n \in \mathbb{N}$ and (20), we get

$$
\begin{aligned}
d(T z, z) \leqslant & d\left(T z, T^{n+1} x_{0}\right)+d\left(T^{n+1} x_{0}, z\right) \\
\leqslant & \varphi\left(d\left(z, T^{n} x_{0}\right)\right)+K\left\{d(z, T z)+d\left(T^{n} x_{0}, T^{n+1} x_{0}\right)\right\} \\
& +d\left(T^{n+1} x_{0}, z\right),
\end{aligned}
$$

So

$(1-K) d(T z, z) \leqslant \varphi\left(d\left(z, T^{n} x_{0}\right)\right)+K d\left(T^{n} x_{0}, T^{n+1} x_{0}\right)+d\left(T^{n+1} x_{0}, z\right) \rightarrow 0$, 
as $n \rightarrow \infty$. In consequence, $d(T z, z)=0$. Hence $z=T z$.

Remark 4.6. We do not know whether in this case with assumptions that for every $(x, y) \in X \times X$ there exists $w \in X$ such that $x \preccurlyeq w$ and $y \preccurlyeq w$ and $K>0$, there is exactly one fixed point. When $K=0$, see Theorem 2.2 or [4, Theorem 3.6 and 3.10].

Attempting to obtain in preordered set results inspired by the recent work of Karapinar et al. [10] encounters serious difficulties and opens the way for further research. Now we present a partial result in this direction.

Theorem 4.7. Let $(X, d, \preccurlyeq)$ be a preordered complete metric space and $T$ : $X \rightarrow X$ be an asymptotically regular and monotone ( $\preccurlyeq$-non-decreasing) mapping and such that $x_{0} \preccurlyeq T x_{0}$ for some $x_{0} \in X$, and

$$
\psi(d(T x, T y)) \leqslant \varphi(d(x, y))+K \cdot\{d(x, T x)+d(y, T y)\}
$$

for any $x, y \in X$ with $x \preccurlyeq y$ and $d(T x, T y)>0$, where $0<K<\infty$ and where the functions $\psi, \varphi:(0, \infty) \rightarrow \mathbb{R}$ satisfy one of the following conditions:

(i) $\lim \sup _{t \rightarrow \varepsilon} \varphi(t)<\liminf _{t \rightarrow \varepsilon+} \psi(t)$ for any $\varepsilon>0$;

(ii) $\lim \sup _{t \rightarrow \varepsilon+} \varphi(t)<\liminf _{t \rightarrow \varepsilon} \psi(t)$ for any $\varepsilon>0$;

(iii) $\lim \sup _{t \rightarrow \varepsilon} \varphi(t)<\psi(\varepsilon+)$ for any $\varepsilon>0$.

Suppose that one of the following conditions holds:

(a) $T$ is $k$-continuous for some $k \geqslant 1$;

(b) $T$ is orbitally continuous.

Then $T$ has a fixed point.

Proof. Following the proof of Theorem 2.2, we have a $\preccurlyeq$-non-decreasing sequence $\left\{x_{n}=T x_{n-1}\right\}, n \geqslant 1$ :

$$
x_{0} \preccurlyeq x_{1} \preccurlyeq x_{2} \preccurlyeq \cdots \preccurlyeq x_{n-1} \preccurlyeq x_{n} \preccurlyeq x_{n+1} \preccurlyeq \cdots
$$

If $x_{n}=x_{n+1}$ for some $n \geqslant 0$, then $x_{n}=T x_{n}$, so $x_{n}$ is the fixed point of $T$.

Let $\psi$ and $\varphi$ satisfy (i). Suppose, $x_{n} \neq x_{n+1}$ for all $n \geqslant 0$. Assume that $\left\{x_{n}\right\}$ is not a Cauchy sequence. It follows from Lemma 2.1 that there exists $\varepsilon>0$ and two subsequences $\left\{x_{n_{k}}\right\}$ and $\left\{x_{m_{k}}\right\}$ of $\left\{x_{n}\right\}$ such that the limits (2) and (3) hold. It follows from (2) that $d\left(x_{n_{k}+1}, x_{m_{k}+1}\right)>\varepsilon>0$ for all $k \geqslant 1$ and exists $k_{0} \geqslant 1$ such that $d\left(x_{n_{k}}, x_{m_{k}}\right)>0$ for all $k \geqslant k_{0}$. Applying (21) with $x=x_{n_{k}}$ and $y=x_{m_{k}}$, we get

$\psi\left(d\left(x_{n_{k}+1}, x_{m_{k}+1}\right)\right) \leqslant \varphi\left(d\left(x_{n_{k}}, x_{m_{k}}\right)\right)+K \cdot\left\{d\left(x_{n_{k}}, x_{n_{k}+1}\right)+d\left(x_{m_{k}}, x_{m_{k}+1}\right)\right\}$

for all $k \geqslant k_{0}$. Taking into account the asymptotic regularity of $T$ and (2) and (3), letting the limit as $k \rightarrow \infty$, we get

$$
\begin{aligned}
\liminf _{t \rightarrow \varepsilon+} \psi(t) & \leqslant \liminf _{k \rightarrow \infty} \psi\left(d\left(x_{n_{k}+1}, x_{m_{k}+1}\right)\right) \\
& \leqslant \limsup _{k \rightarrow \infty} \varphi\left(d\left(x_{n_{k}}, x_{m_{k}}\right)\right) \leqslant \limsup _{t \rightarrow \varepsilon} \varphi(t),
\end{aligned}
$$

which is a contradiction to (i). Therefore, $\left\{x_{n}\right\}$ is a Cauchy sequence. Because $X$ is complete $\lim _{n \rightarrow \infty} x_{n}=z \in X$.

Let $\psi$ and $\varphi$ satisfy (ii). Suppose, $x_{n} \neq x_{n+1}$ for all $n \geqslant 0$. Assume that $\left\{x_{n}\right\}$ is not a Cauchy sequence. Same as in Lemma 2.1 [15, Lemma 2.2] 
there exists $\varepsilon>0$ and two subsequences $\left\{x_{n_{k}}\right\}$ and $\left\{x_{m_{k}}\right\}$ of $\left\{x_{n}\right\}$ such that $\lim _{k \rightarrow \infty} d\left(x_{n_{k}+1}, x_{m_{k}+1}\right)=\varepsilon$ and $\lim _{k \rightarrow \infty} d\left(x_{n_{k}}, x_{m_{k}}\right)=\varepsilon+$. It follows from this that $d\left(x_{n_{k}}, x_{m_{k}}\right)>\varepsilon>0$ for all $k \geqslant 1$ and there exists $k_{0} \geqslant 1$ such that $d\left(x_{n_{k}+1}, x_{m_{k}+1}\right)>0$ for all $k \geqslant k_{0}$. Applying (21) with $x=x_{n_{k}}$ and $y=x_{m_{k}}$, we get

$\psi\left(d\left(x_{n_{k}+1}, x_{m_{k}+1}\right)\right) \leqslant \varphi\left(d\left(x_{n_{k}}, x_{m_{k}}\right)\right)+K \cdot\left\{d\left(x_{n_{k}}, x_{n_{k}+1}\right)+d\left(x_{m_{k}}, x_{m_{k}+1}\right)\right\}$

for all $k \geqslant k_{0}$. Taking into account the asymptotic regularity of $T$ and letting the limit as $k \rightarrow \infty$, we get

$$
\begin{aligned}
\liminf _{t \rightarrow \varepsilon} \psi(t) & \leqslant \liminf _{k \rightarrow \infty} \psi\left(d\left(x_{n_{k}+1}, x_{m_{k}+1}\right)\right) \\
& \leqslant \limsup _{k \rightarrow \infty} \varphi\left(d\left(x_{n_{k}}, x_{m_{k}}\right)\right) \leqslant \limsup _{t \rightarrow \varepsilon+} \varphi(t),
\end{aligned}
$$

which is a contradiction to (ii). Therefore, $\left\{x_{n}\right\}$ is a Cauchy sequence. Because $X$ is complete $\lim _{n \rightarrow \infty} x_{n}=z \in X$.

Let $\psi$ and $\varphi$ satisfy (iii). Suppose, $x_{n} \neq x_{n+1}$ for all $n \geqslant 0$. Assume that $\left\{x_{n}\right\}$ is not a Cauchy sequence. It follows from Lemma 2.1 that there exists $\varepsilon>0$ and two subsequences $\left\{x_{n_{k}}\right\}$ and $\left\{x_{m_{k}}\right\}$ of $\left\{x_{n}\right\}$ such that the limits (2) and (3) hold. It follows from (2) that $d\left(x_{n_{k}+1}, x_{m_{k}+1}\right)>\varepsilon>0$ for all $k \geqslant 1$ and exists $k_{0} \geqslant 1$ such that $d\left(x_{n_{k}}, x_{m_{k}}\right)>0$ for all $k \geqslant k_{0}$. Applying (21) with $x=x_{n_{k}}$ and $y=x_{m_{k}}$, we get

$\psi\left(d\left(x_{n_{k}+1}, x_{m_{k}+1}\right)\right) \leqslant \varphi\left(d\left(x_{n_{k}}, x_{m_{k}}\right)\right)+K \cdot\left\{d\left(x_{n_{k}}, x_{n_{k}+1}\right)+d\left(x_{m_{k}}, x_{m_{k}+1}\right)\right\}$ for all $k \geqslant k_{0}$. Taking into account the asymptotic regularity of $T$ and (2) and (3), letting the limit as $k \rightarrow \infty$, we get

$$
\begin{aligned}
\psi(\varepsilon+) & =\lim _{k \rightarrow \infty} \psi\left(d\left(x_{n_{k}+1}, x_{m_{k}+1}\right)\right. \\
& \leqslant \limsup _{k \rightarrow \infty} \varphi\left(d\left(x_{n_{k}}, x_{m_{k}}\right)\right) \leqslant \limsup _{t \rightarrow \varepsilon} \varphi(t),
\end{aligned}
$$

which is a contradiction to (iii). Therefore, $\left\{x_{n}\right\}$ is a Cauchy sequence. Because $X$ is complete $\lim _{n \rightarrow \infty} x_{n}=z \in X$.

Analogically as in the proof of Theorem 2.2, $z$ is a fixed point of $T$.

We will now give an example of a mapping that satisfy (20) and (21) with $\varphi(t)=\frac{t}{1+t}$ and $\psi(t)=t, t>0$.

Example 4.8. Let $X=[0,1]$ be with the usual metric. Define $T: X \rightarrow X$ by $T x=\frac{x}{1+x}$ if $x \in[0,1)$ and $T 1=\frac{3}{4}$. Obviously $T$ is not a Lipschitz mapping. If $x=\frac{3}{4}$ and $y=1$, then

$|T x-T y|=\frac{9}{28}=\max \left\{|x-y|,|x-T x|,|y-T y|, \frac{1}{2}\{|x-T y|+|y-T x|\}\right\}$,

so $T$ does not satisfy Ćirić's condition. Next we observe there is no constant $0 \leqslant M<1$ and $K \in[0, \infty)$ such that for all $x, y \in X$ satisfying $|T x-T y| \leqslant$ $M \cdot|x-y|+K \cdot\{|x-T x|+|y-T y|\}$. Indeed, if $x=0$ and $y=\varepsilon>0$ (sufficiently small), then $|T 0-T \varepsilon|=\frac{\varepsilon}{1+\varepsilon} \leqslant M \cdot \varepsilon+K \cdot\left(\varepsilon-\frac{\varepsilon}{1+\varepsilon}\right)$, so $M+K \geqslant \frac{1-M}{\varepsilon} \rightarrow \infty$ as $\varepsilon \downarrow 0$.

On the other hand, $T$ satisfy $|T x-T y| \leqslant \varphi(|x-y|)$ with $\varphi(t)=\frac{t}{1+t}$ if $x, y \in[0,1)$. Note that if $x=\frac{3}{4}$ and $y=1$, then $\left|T\left(\frac{3}{4}\right)-T 1\right|=\frac{9}{28}>\frac{1}{5}=\varphi\left(\frac{1}{4}\right)$. 
For $x=0$ and $y=1,|T 0-T 1|=\frac{3}{4}=\varphi(1)+\{|0-T 0|+|1-T 1|\}$, and easy calculations (for $0<x<1$ and $y=1$ ) show that the mapping $T$ satisfies

$$
|T x-T y| \leqslant \varphi(|x-y|)+\{|x-T x|+|y-T y|\}
$$

for all $x, y \in[0,1]$.

Open Access. This article is licensed under a Creative Commons Attribution 4.0 International License, which permits use, sharing, adaptation, distribution and reproduction in any medium or format, as long as you give appropriate credit to the original author(s) and the source, provide a link to the Creative Commons licence, and indicate if changes were made. The images or other third party material in this article are included in the article's Creative Commons licence, unless indicated otherwise in a credit line to the material. If material is not included in the article's Creative Commons licence and your intended use is not permitted by statutory regulation or exceeds the permitted use, you will need to obtain permission directly from the copyright holder. To view a copy of this licence, visit http:// creativecommons.org/licenses/by/4.0/.

Publisher's Note Springer Nature remains neutral with regard to jurisdictional claims in published maps and institutional affiliations.

\section{References}

[1] Boyd, D.W., Wong, J.S.W.: On nonlinear contractions. Proc. Am. Math. Soc. 20(2), 458-464 (1969)

[2] Ćirić, L.: On contraction type mappings. Math. Balkanica 1, 52-57 (1971)

[3] Geraghty, M.A.: On contractive mappings. Proc. Am. Math. Soc. 40(2), 604608 (1973)

[4] Górnicki, J.: Remarks on contractive type mappings. Fixed Point Theory Appl. 2017, 8 (2017)

[5] Górnicki, J.: Fixed point theorems for Kannan type mappings. J. Fixed Point Theory Appl. 19, 2145-2152 (2017)

[6] Górnicki, J.: Remarks on asymptotic regularity and fixed points. J. Fixed Point Theory Appl. 21, 29 (2019)

[7] Górnicki, J.: On some mappings with a unique fixed point. J. Fixed Point Theory Appl. 22, 8 (2020)

[8] Hitzler, P., Seda, A.K.: Dislocated topologies. J. Electr. Eng. 51(12/s), 3-7 (2000)

[9] Kannan, R.: Some results on fixed points. Bull. Calcutta Math. Soc. 60, 71-76 (1968)

[10] Karapınar, E., De La Sen, M., Fulga, A.: A note on the Górnicki-Proinov type contractions. J. Funct. Spaces (2021). https://doi.org/10.1155/2021/6686644. ((article ID 6686644, 8 pages $)$ )

[11] Nieto, J.J., Rodríguez-López, R.: Contractive mapping theorems in partially ordered sets and applications to ordinary differential equations. Order 22, 223$239(2005)$ 
[12] O'Regan, D., Petruşel, A.: Fixed point theorems for generalized contractions in ordered metric spaces. J. Math. Anal. Appl. 341, 1241-1252 (2008)

[13] Pant, A., Pant, R.P.: Fixed points and continuity of contractive maps. Filomat 31(11), 3501-3506 (2017)

[14] Proinov, P.D.: Fixed point theorems in metric spaces. Nonlinear Anal. 64, 546557 (2006)

[15] Proinov, P.D.: Fixed point theorems for generalized contractive mappings in metric spaces. J. Fixed Point Theory Appl. 22, 21 (2020)

[16] Ran, A.C.M., Reurings, M.C.B.: A fixed point theorem in partially ordered sets and some applications to matrix equations. Proc. Am. Math. Soc. 132, 1435-1443 (2003)

[17] Reich, S.: Kannan's fixed point theorem. Boll. Un. Mat. Ital. (4) 4, 1-11 (1971)

[18] Skof, F.: Teoremi di punto fisso per applicazioni negli spazi metrici. Atti. Acad. Sci. Torino Cl. Sci. Fis. Mat. Natur. 111, 323-329 (1977)

\author{
Jarosław Górnicki \\ Department of Mathematics and Applied Physics \\ Rzeszów University of Technology \\ P.O. Box 85 35-959 Rzeszów \\ Poland \\ e-mail: gornicki@prz.edu.pl
}

Accepted: October 5, 2021. 